\title{
Pattern Directed Phase Transitions and VOC Sensing of Liquid Crystal Films
}

Bolleddu Ravi, ${ }^{a}$ Snigdha Chakraborty, ${ }^{\text {a }}$ Mitradip Bhattacharjee, ${ }^{c}$ Nandini Bhandaru, ${ }^{\text {b Siddharth }}$ Thakur, ${ }^{\mathrm{a}}$ Partho Sarathi Gooh-Pattader, ${ }^{\mathrm{a}, \mathrm{c}}$ Rabibrata Mukherjee, ${ }^{\mathrm{b}}$ and Dipankar Bandyopadhyay*a,c

aDepartment of Chemical Engineering, Indian Institute of Technology Guwahati, Assam, 781039, India

${ }^{b}$ Department of Chemical Engineering, Indian Institute of Technology Kharagpur, West Bengal, 721302, India

${ }^{c}$ Centre for Nanotechnology, Indian Institute of Technology Guwahati, Assam, 781039, India

\footnotetext{
*Corresponding author. Dipankar Bandyopadhyay, Centre for Nanotechnology and Department of Chemical Engineering, Indian Institute of Technology Guwahati, 781039 - India. Email: dipban@iitg.ernet.in
} 


\section{A. Substrate Preparation}

The Si substrates of dimension $1.5 \mathrm{~cm} \times 1.5 \mathrm{~cm}$ were cut from the main wafer and were then cleaned according to the following protocol. Initially, the substrates were subjected to sonication for $30 \mathrm{~min}$ in a bath containing SDS solution. Then, after washing with copious amounts of DI water, the substrates were immersed in boiling solutions of methanol and acetone for 15 min each. Thereafter, they were put in a bath containing definite proportions of $\mathrm{H}_{2} \mathrm{O}_{2}, \mathrm{NH}_{3}$ and DI water and the solution was allowed to reach boiling point where it was kept for $30 \mathrm{~min}$. After allowing it to cool down, the substrates were again washed with DI water and subsequently dried in the presence of $\mathrm{N}_{2}$ gas. After the substrates were ready, $5 \%(\mathrm{w} / \mathrm{v})$ concentration of PS solution in toluene was spin coated at $2500 \mathrm{rpm}$ for $120 \mathrm{~s}$. The films were dried in vacuum oven at $60^{\circ} \mathrm{C}$ for $24 \mathrm{~h}$ to remove the excess solvent.

The stripe patterned PDMS master stamp was prepared by pouring PDMS on compact disk. ${ }^{1} \mathrm{~A}$ small size of the stamp was cut and immersed in toluene for $30 \mathrm{~s}$ before placing it on the PS film for 2 min. Thereafter, in order to transfer the PDMS patterns, the PS film was annealed through the solvent vapor exposure. For this reason, the PS film with the PDMS stamp on the top was kept in a Petri dish for $2-3$ min along with $8-10$ solvent chambers filled with toluene. Under this condition, the PS film developed negative replica of PDMS master stamp due to capillary force lithography (CFL). ${ }^{2}$ Following this, after $2-3 \mathrm{~min}$ of solvent exposure the PS film was isolated and dried in ambient condition. The surface pattern was analyzed by atomic force microscopy (AFM, Innova Iris, Bruker-Icon Analytical Equipment). The dimensions of the stripes on the PS surface were $800 \mathrm{~nm}$ width, $120 \mathrm{~nm}$ deep, and periodicity of $1.6 \mu \mathrm{m}$, as shown in the Figure $1 \mathrm{~B}$ of the main manuscript. The box patterned PS surface with $10 \mu \mathrm{m}$ width, $300 \mathrm{~nm}$ depth, and $20 \mu \mathrm{m}$ periodicity, as shown in the Figure $1 \mathrm{C}$ was fabricated by a modified pressure assisted imprinting technique, the details of which can be found elsewhere. ${ }^{3}$

In order to prepare the chemically patterned surface, initially, an OTS monolayer was deposited on the thoroughly cleaned silicon wafers by immersing them in OTS solution ( $5 \mu$ OTS in $5 \mathrm{ml}$ bicyclohexane) for $20 \mathrm{~min}$. Following this, the substrates were sonicated in chloroform for $20 \mathrm{~min}$ then dried in the stream of $\mathrm{N}_{2} \cdot{ }^{4}$ Thereafter, the OTS coated silicon substrates were masked by a TEM grid before they were exposed to UV-Ozone (Novascan Tech. Inc., USA) for $2 \mathrm{~h}$. The UV passed through the "box" openings of the TEM grid to etch out the OTS layer while under the $\mathrm{Cu}$ grid/bar the OTS film remained as such. Thus, the UV-Ozone treatment led to the formation of 
$\mathrm{Si} / \mathrm{SiO}_{2}$ "box" patterns and OTS "grid" patterns on the Si wafer, as shown in the Figure 1(D). In order to study the pattern directed phase transitions, the 5CB films ( $240 \%(\mathrm{w} / \mathrm{v})$ in hexane) were initially spin coated on the surfaces with physical and chemical patterns, as shown Figure 1. The thicker films were coated to avoid the issues associated with the spin-dewetting for the thinner films. Following this, the solvent vapor experiments were conducted with hexane as mentioned previously.

\section{B. Effect of different concentrations of $5 \mathrm{CB}$ film on phase transition time period}

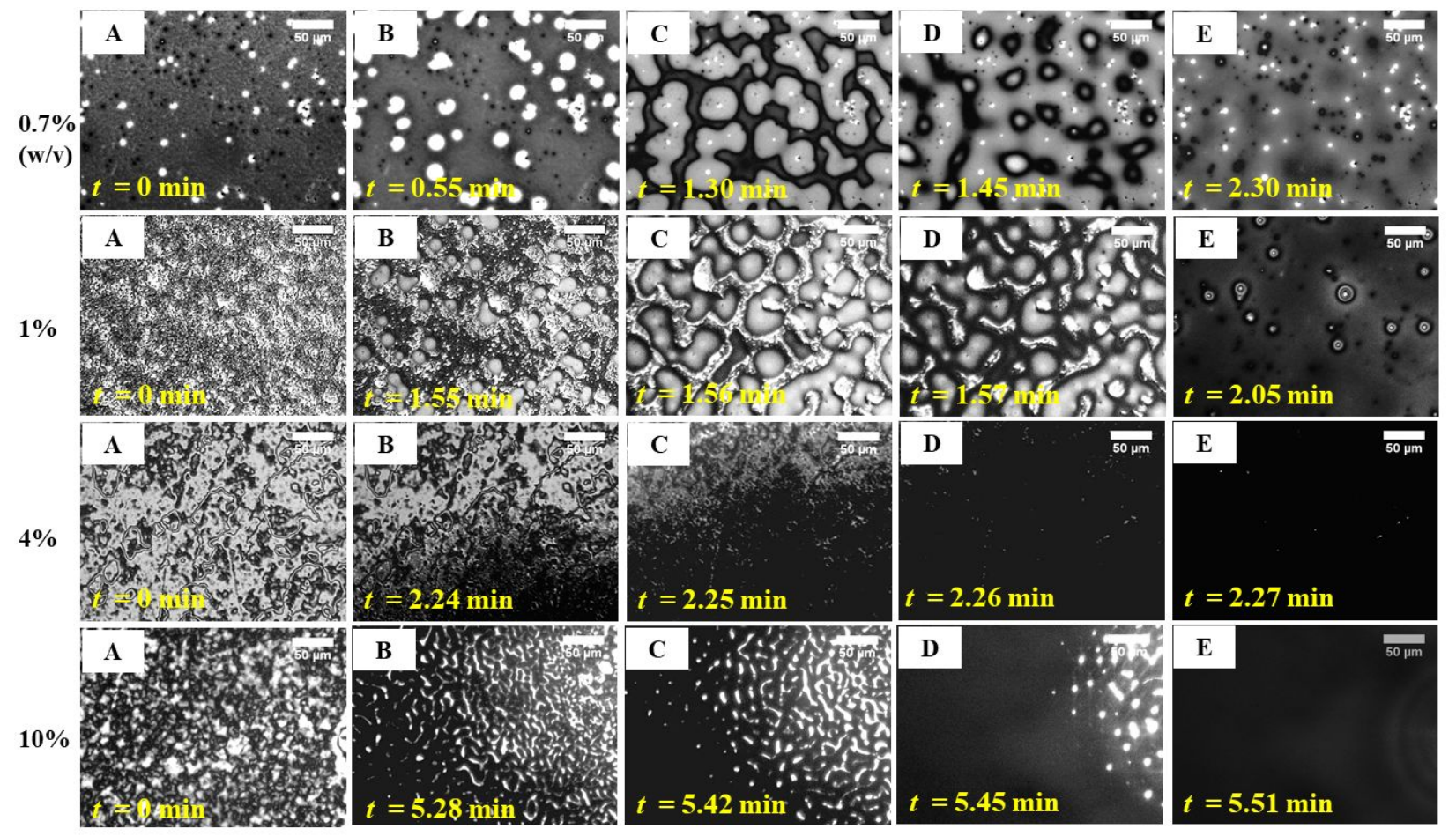

Figure S1. Cross-polarized optical micrographs of the solvent vapor annealed phase transition of 5CB films of different concentrations in hexane, at ambient temperature $\left(\mathrm{T}=24 \pm 1^{\circ} \mathrm{C}\right)$. Images $(\mathrm{A}-\mathrm{E})$ in row I denotes the nematic to isotropic phase transition in a $0.7 \%(\mathrm{w} / \mathrm{v}) \mathrm{LC}$ film, whereas in row II, III and IV, they denote phase transitions in $1 \%(\mathrm{w} / \mathrm{v}), 4 \%(\mathrm{w} / \mathrm{v})$ and $10 \%(\mathrm{w} / \mathrm{v})$ LC films, respectively.

Different films of 5CB in hexane were prepared following the similar procedure as reported in methods section. 5CB concentration in the films ranged from $0.7 \%(\mathrm{w} / \mathrm{v})$ to $10 \%(\mathrm{w} / \mathrm{v})$. The thickness of the films varied from $\sim 74 \mathrm{~nm}$ from $0.7 \%(\mathrm{w} / \mathrm{v}), \sim 100 \mathrm{~nm}$ for $1 \%(\mathrm{w} / \mathrm{v})$ to few $\mu \mathrm{m}$ for higher concentrations. Figure S1 shows the variation in the phase transition time observed for liquid crystal films of varying concentrations. It can be observed that the time required for the first spot to appear (i.e. the initial moment when the conversion of nematic order to isotropic took place) 
increased with increasing film thickness. This could be explained by understanding that increasing film thickness offered higher diffusional resistance to the incoming vapors and hence a longer time period was required for their uptake within the liquid crystal system. However, once the nematic to isotropic phase change took place at a particular location, the time required for the phase transition within the entire liquid crystal film matrix decreased with increasing film thickness. For liquid crystal films with smaller thickness values, the orientation of molecules at the free surface is significantly affected by the strongly anchored molecules at the substrate. For a thicker film however, since the molecules at the free interface are relatively far away from the substrate, the change in the phase transition requires considerably less time. ${ }^{5}$

\section{Descriptions of Videos}

Supporting video 1: This video shows solvent vapor mediated phase transitions of crystalline phase 7CB liquid crystal. Initially, when the ultrathin $(h=51 \pm 1 \mathrm{~nm})$ film was exposed to solvent vapor then the film changed its phase from crystal to nematic after, $t=93 \mathrm{~s}$. This can be followed in the video with the reduction in the intensity of reflected light from the film surface. Following this, a nematic to isotropic (NI) was observed as more solvent vapor diffused into the film. The NI phase transition was indicated by the appearance of isotropic gray patches on film surface after, $t$ $=99 \mathrm{~s}$. Later, they grew in size at $t=102 \mathrm{~s}$ before coalescing to form bi-continuous surface patterns at, $t=105 \mathrm{~s}$. Finally, the phase transition ended with by the disappearance of the surface patterns at, $t=116 \mathrm{~s}$. At this stage, when the solvent exposure was withdrawn, the film came back to its original phase at room temperature. During this process, the nematic textures appeared at patches on the film surface at, $t=43 \mathrm{~s}$. They grew in size until, $t=45 \mathrm{~s}$, before forming a bi-continuous pattern after, $t=49 \mathrm{~s}$, and converted into nematic state after, $t=56 \mathrm{~s}$. Finally, the entire process finished after the transition from nematic to crystal state was complete at, $t=63 \mathrm{~s}$.

Supporting video 2: This video shows solvent vapor mediated phase transitions of smectic $8 \mathrm{CB}$ liquid crystal. When the solvent vapor was exposed to smectic film $(h=54 \pm 1 \mathrm{~nm})$ with a mosaic texture on the surface, the film would change its phase from smectic to nematic after, $t=36 \mathrm{~s}$, indicated by the destruction of the mosaic texture. After this, when the solvent exposure was extended further, the nematic film changed to isotropic state at, $t=75 \mathrm{~s}$. At this stage, when the 
solvent exposure was withdrawn, the isotropic film again changed to nematic state after, $t=14 \mathrm{~s}$ and then from nematic to smectic state after, $t=38 \mathrm{~s}$.

Supporting video 3: This video shows temperature induced phase transitions of smectic state $8 \mathrm{CB}$ liquid crystal ultrathin film $(h=54 \pm 1 \mathrm{~nm})$. The smectic film having mosaic surface texture changed its phase from smectic to nematic when $T=30.1 \pm 0.5^{\circ} \mathrm{C}$, indicated by the destruction of mosaic texture along with attaining nematic texture. Thereafter, nematic film changed its phase from nematic to isotropic state at $T=38.3 \pm 0.5^{\circ} \mathrm{C}$. Following this, when the temperature was decreased, the film would change its phase from isotropic to nematic state (at, $\mathrm{T}=38.3 \pm 0.5^{\circ} \mathrm{C}$ ) before attaining the smectic state $\left(\right.$ at, $\left.\mathrm{T}=30.1 \pm 0.5^{\circ} \mathrm{C}\right)$ at room temperature.

Supporting video 4: This video shows solvent vapor induced phase transitions of LC drop, which was placed in between a pair of electrodes. The variation in the electrical resistance with the phase transition of the drop has been recorded and shown on the multimeter. When the solvent vapor was exposed to the LC drop, the NI transition led a reduction in the electrical resistance, as shown on the multimeter. The video shows that at the isotropic state the electrical resistance of the drop reached a minimum value. At this stage, when the solvent vapor exposure was withdrawn, the electrical resistance increased again as the drop underwent IN phase transition. Finally, the resistance reached the initial value after the drop attained its LC state.

Supporting video 5: The video shows that a LC film resting on a physically heterogeneous surface with periodic stripe patterns could undergo a pattern-directed phase transition. The video suggests that the pattern-directed phase transitions could be observed when the films were thinner (e.g. LC concentration less than $5 \%(\mathrm{w} / \mathrm{v})$ in the solvent while spin-casting). However, the effect of the underlying substrate on the phase transition decreased with increasing LC film thickness (e.g. LC concentration more than $10 \%(\mathrm{w} / \mathrm{v})$ in the solvent while spin-casting). Finally, there was no effect of the underlying substrate patterns on the phase transition when the LC films were very thick (e.g. LC concentration $40 \%(\mathrm{w} / \mathrm{v})$ in the solvent while spin-casting).

Supporting video 6: The video shows the pattern-directed phase transition of a LC film resting on a physically heterogeneous surface with an array of periodic box-patterns. The video suggests 
that the pattern-directed phase transitions could be observed when the films were thinner (e.g. LC concentration less than $3 \%(\mathrm{w} / \mathrm{v})$ in the solvent while spin-casting). However, the effect of the underlying substrate decreased with increasing LC film thickness (e.g. LC concentration more than $6 \%(\mathrm{w} / \mathrm{v})$ in the solvent while spin-casting). Finally, there was no effect of the underlying substrate patterns on the phase transitions of the LC films of high thickness (e.g. LC concentration more than $10 \%(\mathrm{w} / \mathrm{v})$ in the solvent while spin-casting).

Supporting video 7: This video shows the phase transition of a LC film resting on a chemically heterogeneous substrate with periodic box shaped hydrophilic patches surrounded by grid shaped hydrophobic patches. The video shows that when the solvent was exposed to LC film, the NI phase transition occurred faster on the grid shaped hydrophobic patches before the same took place on the box shaped hydrophilic patches. However, when the solvent vapor exposure was withdrawn, the phase transition was faster on the hydrophobic patches while the same took place later on the hydrophilic ones.

\section{References}

(1) Mukherjee, R.; Sharma, A.; Gonuguntla, M.; Patil, G. K. Adhesive Force Assisted Imprinting of Soft Solid Polymer Films by Flexible Foils. J Nanosci. Nanotechnol., 2008, 8, 3406.

(2) Suh, K.Y.; Kim, Y. S.; Lee, H.H. Capillary Force Lithography. Adv. Mater., 2001, 13, 1386.

(3) Bhandaru, N.; Das, A.; Mukherjee, R. Confinement induced ordering in dewetting of ultrathin polymer bilayers on nanopatterned substrates. Nanoscale, 2016, 8, 1073.

(4) Herzer, N.; Wienk, M. M.; Schmit, P.; Spoelstra, A. B.; Hendriks, C. E.; Oosterhout, S. D.; Hoeppener, S.; Schubert, U. S. Fabrication of PEDOT-OTS-patterned ITO substrates. J. Mater. Chem., 2010, 20, 6618.

(5) Ravi, B.; Mukherjee, R.; Bandyopadhyay, D. Solvent vapour mediated spontaneous healing of self-organized defects of liquid crystal films. Soft Matter, 2015, 11, 139. 